\title{
A MICROFABRICATED WIRELESS RF PRESSURE SENSOR MADE COMPLETELY OF BIODEGRADABLE MATERIALS
}

\author{
Mengdi Luo ${ }^{1 *}$, Chao Song ${ }^{2}$, Florian Herrault ${ }^{2}$ and Mark G. Allen ${ }^{2}$
}

${ }^{1}$ School of Materials Science and Engineering, Georgia Institute of Technology, Atlanta, Georgia, USA

${ }^{2}$ School of Electrical and Computer Engineering, Georgia Institute of Technology, Atlanta, Georgia, USA

\begin{abstract}
Implantable passive LC resonant sensors have previously been studied to wirelessly measure physiological parameters in vivo. In this work, a wireless RF pressure sensor made entirely of biodegradable materials is designed and fabricated via MEMS technologies. Such biodegradable implants may be appropriate for short-term, acute medical applications as they potentially eliminate the need for implant extraction when sensing is no longer required. Passive LC resonant sensors typically require conductors, structural materials, and dielectrics to function. Metallic zinc, known to be biocompatible, was also found to be degradable in saline and was subsequently used as the sensor conductor material, while biodegradable polymers were used as dielectrics and structural materials. Biodegradable materials are particularly challenging to process using conventional MEMS fabrication techniques because they are water-sensitive. As a result, alternative MEMS fabrication approaches using embossing and multi-layer folding were combined with traditional techniques to fabricate the pressure sensor. Experimentally, the fabricated sensor made of biodegradable materials demonstrated linear frequency response with external applied pressure. A sensitivity of $-290 \mathrm{kHz} / \mathrm{kPa}$ was measured in the $0-30 \mathrm{kPa}$ pressure range.
\end{abstract}

\section{INTRODUCTION}

Implantable resonant passive sensors were proposed more than five decades ago as a solution to wirelessly measure physical parameters within living organisms [1]. Since then, non-degradable MEMS-fabricated passive resonant sensors have been demonstrated for chronic implantable applications to monitor blood pressure and other physiological conditions [2]. Although such sensors show great promise for chronic applications, some acute or shorter-term medical applications (e.g., wound or bone healing) could also benefit from implantable monitoring sensors. Typically these sensors should be extracted when no longer needed. In these applications, if instead the sensor could be made completely biodegradable, this extraction could be avoided.

A number of researchers have proposed approaches to implantable biodegradable sensors. Wireless implantable strain sensors consisting of a micro-channel attached to a fluid reservoir were proposed in [3]. These devices utilized an external ultrasound system to assess the strain-driven filling of the channel, which could be limiting compared to RF readout. Biodegradable polymeric composite materials that could have application as components of RLC resonant sensors, prepared by emulsion polymerization of pyrrole in biodegradable polylactic acid (PLA) and polycaprolactone (PCL) polymers, are also under study [4]. Non-sensing implantable biodegradable stimulators that exploit RF coupling have also been proposed as an approach to promote bone growth. The coupling coil was fabricated using conventional micromilling of biodegradable magnesium alloys, and packaged with poly L:D lactide polymer [5].

The microfabricated pressure sensor presented in this work is also based on the passive resonant mechanism [2]. It utilizes RF interrogation while simultaneously maintaining its all-biodegradable character. The pressure sensor consists of a sensing cavity, bounded by two electrodes forming the variable capacitor and connected in series with an inductor coil (Figure 1). The inductor coil not only acts as an essential component of the resonant sensor, but also provides means for magnetically coupling the sensor to an external (e.g., outside the body) coil. When pressure is applied to the sensor, the gap between the two capacitive electrodes is reduced and the capacitor value increases. The resulting pressure-induced change in the LC resonant frequency can be measured wirelessly using the external coil.
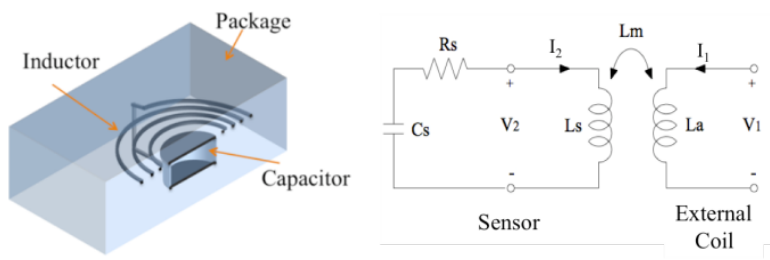

Figure 1: Schematic cross-section diagram (left) and equivalent circuit (right) of a passive $L C$ resonant sensor.

\section{DESIGN AND FABRICATION Materials}

There are several FDA-approved and commercially-available biocompatible and biodegradable polymers [6]. Some design constraints that may drive polymer choice include desired degradation time, processing/fabrication characteristics, and mechanical properties. PLA and PCL polymers have been selected in this application. PLA is used as the packaging and sensing plate material because of its mechanical properties [6], and PCL is utilized as the bonding and sealing material because of its low melting temperature (approximately $61^{\circ} \mathrm{C}$ ), which is compatible with the proposed fabrication process. Other potential biodegradable and biocompatible polymers include copolymers of PLA with polyglycolic acid (PLGA) and polyanhydrides. By varying the biodegradable polymer selection, different desired degradation times ranging from 3-24 months can be obtained [6].

The most well-known biocompatible and biodegradable metals are magnesium and iron. These metals can degrade within the body to form soluble species. Further, they have been demonstrated in biodegradable stent applications [7]. However, from a MEMS fabrication standpoint, standard approaches to create thick micro-scale conductors for high quality factor LC resonant devices, such as electrodeposition, are challenging with active elements such as magnesium. Iron is more compatible with standard electrodeposition [8], but at higher frequencies (e.g., tens of $\mathrm{MHz}$ and above), its $\mathrm{AC}$ conductivity is poor because of the relative magnetic permeability enhancement of the skin effect [9].

Zinc is considered one of the biocompatible metals and is also an essential element consumed by the human body [10]. Unlike iron, its AC electrical characteristics should enable the design and fabrication of high quality factor inductors (its bulk resistivity is only 3.5 times higher than that of copper). Zinc is also compatible with electroplating technology, making it a good candidate as a conductive material in microfabricated LC resonant biodegradable pressure sensor applications. 


\section{Device Design}

The operating frequency of passive wireless devices is typically bounded by maximum device size (at low frequencies) and signal degradation in the lossy medium of the body (at high frequencies). It has been demonstrated that operating frequencies in the $10-100 \mathrm{MHz}$ range are suitable for in vivo wireless measurements [2]. To achieve such resonant frequencies while maintaining the device area below a nominal value of $1 \mathrm{~cm}^{2}$, both the inductor and capacitor must follow specific design guidelines. Table 1 shows the design parameters of the RF pressure sensor.

Table 1: Dimensions of the conductors of the pressure sensor

\begin{tabular}{|c|c|c|c|c|c|}
\hline \multicolumn{3}{|c|}{ Zn Inductor } & \multicolumn{3}{c|}{ Zn Capacitor } \\
\hline $\begin{array}{c}\text { Number of } \\
\text { turns }\end{array}$ & $\begin{array}{c}\text { Line } \\
\text { width }\end{array}$ & $\begin{array}{c}\text { Spacing } \\
\text { width }\end{array}$ & $\begin{array}{c}\text { Gap } \\
\text { distance }\end{array}$ & $\begin{array}{c}\text { Pad } \\
\text { radius }\end{array}$ & $\begin{array}{c}\text { Pad } \\
\text { thickness }\end{array}$ \\
\hline & $(\mu \mathrm{m})$ & $(\mu \mathrm{m})$ & $(\mu \mathrm{m})$ & $(\mathrm{mm})$ & $(\mu \mathrm{m})$ \\
\hline 14 & 70 & 70 & 40 & 2.5 & 60 \\
\hline
\end{tabular}

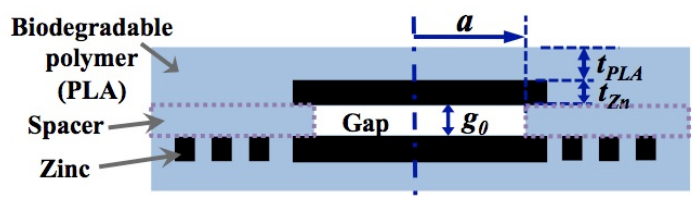

Figure 2: Schematic cross-section of the pressure sensor design.

A schematic cross-section of the pressure sensor is shown in Figure 2. The device consists of a bottom PLA layer with electroplated zinc inductor and capacitor plate, a PLA/PCL spacer layer that forms the sensor cavity, and a top PLA/Zn layer that includes the top capacitor plate. The zinc electrical interconnects between the inductor and the capacitor plates are not depicted. A first-order analytical model indicates that the center deflection of the plates varies linearly with pressure when the gap between the plates is small compared to their thicknesses. The deflection is inversely proportional to the flexural rigidity, which is related to the Young's modulus $E$ and the Poisson's ratio $v$ of the material. Finite element simulations (COMSOL 4.2) coupling electrical with mechanical analyses were performed to evaluate the suitability of the proposed sensor design. A two-dimensional axisymmetric model was used. The geometry of the simulation was simplified by neglecting the zinc coil and via structures. $E$ and $v$ of PLA as well as $\mathrm{Zn}$ were assumed to be $2 \mathrm{GPa}, 0.35$ [9] and $100 \mathrm{GPa}, 0.25$, respectively. Sensors with different thicknesses of PLA $t_{P L A}$ and radii of the deflecting diaphragm $a$ were simulated, with the dimensions shown in Table 2. The initial gap between capacitor plates was set at $40 \mu \mathrm{m}$.

Table 2: Dimensions of the sensor designs for simulation

\begin{tabular}{|c|c|c|c|}
\hline \multirow{2}{*}{ Design } & $\mathrm{a}$ & $\mathrm{t}_{\mathrm{PLA}}$ & $\mathrm{t}_{\mathrm{Zn}}$ \\
\cline { 2 - 4 } & $(\mathrm{mm})$ & $(\mu \mathrm{m})$ & $(\mu \mathrm{m})$ \\
\hline 1 & 2.4 & 200 & 60 \\
\hline 2 & 2.4 & 400 & 60 \\
\hline 3 & 4.9 & 400 & 60 \\
\hline
\end{tabular}

The simulated normalized capacitance change of the simplified sensor structure is presented in Figure 3. Applied pressures ranging from 0 to $30 \mathrm{kPa}$ were simulated. When radius of the cavity $a$ is $2.4 \mathrm{~mm}$ which is smaller than the radius of the $\mathrm{Zn}$ pad $r_{Z n}(2.5 \mathrm{~mm})$, the deflecting diaphragm consists of a Zn/PLA bi-layer plate. Although small, the capacitance change of this sensor would be detectable. Further, the thinner PLA sheet (Design 1) exhibits a higher capacitance change than the thicker PLA sheet (Design 2) for the same cavity radius because of larger deflection. The capacitance variation increases substantially for the sensor with $a=4.9 \mathrm{~mm}$ (Design 3), not only because the deflection radius is increased, but also because the deflecting diaphragm consists of a $\mathrm{Zn} / \mathrm{PLA}$ bi-layer plate embedded in a PLA ring with lower Young's modulus than the bi-layer itself. Experimentally, the deflection of the bottom plate with the zinc coils is expected to be lower than the simulated case because the coil stiffens the bottom layer.

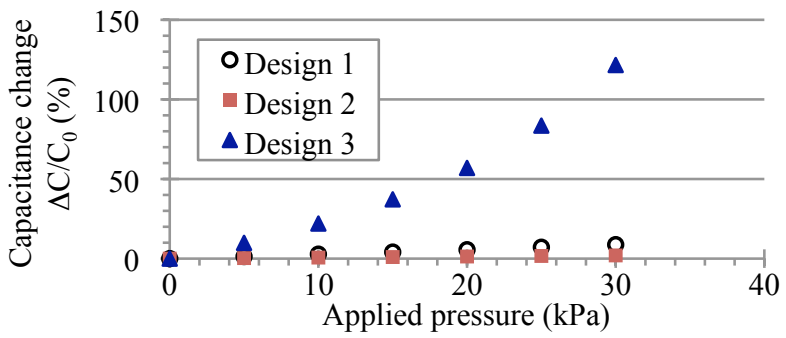

Figure 3: Normalized simulated capacitance change $\Delta C / C_{0}$ as function of external applied pressure on simplified sensor structure for different cavity dimensions and PLA thicknesses.

\section{Pressure Sensor Fabrication}

The fabrication process of the microfabricated RF pressure sensor made of biodegradable materials is schematically shown in Figure 4. Alternative fabrication approaches have been developed to augment conventional approaches since biodegradable materials may be sensitive to chemicals used in standard microfabrication techniques. The process relies on zinc electroplating followed by polymer embossing. A folding technique coupled with polymer lamination steps were utilized to create the sensor cavity and the appropriate LC resonant electrical configuration while alleviating the need for multiple electrodeposition steps.

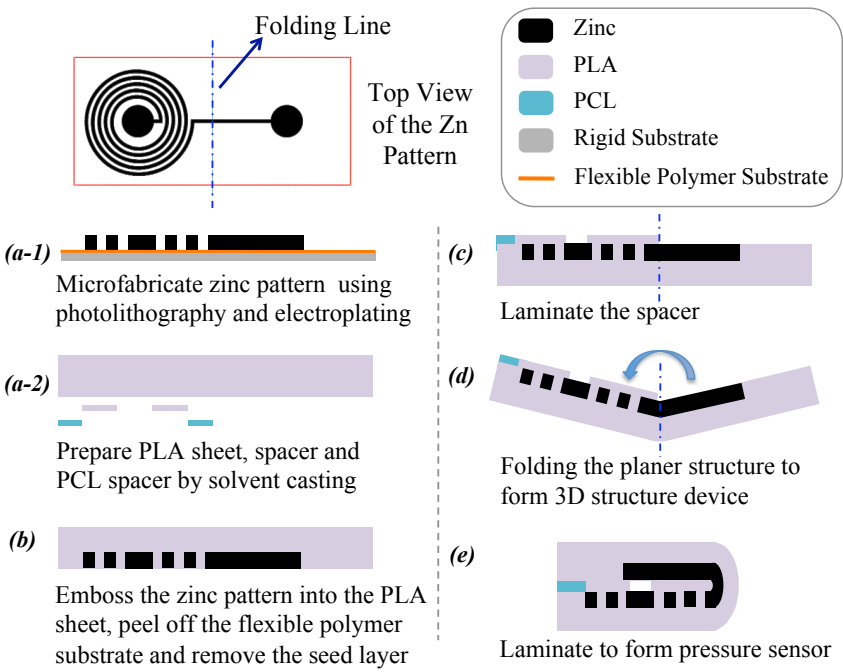

Figure 4: Fabrication process of the passive pressure sensor completely made of biodegradable materials.

The process started with the through-photoresist electrodeposition of zinc patterns (inductor and capacitor plates) onto a flexible substrate. More specifically, an $80-\mu \mathrm{m}$-thick flexible and chemically-resistant polymer film (Kapton ${ }^{\circledR}$ ) with a titanium/copper/titanium seed layer was temporarily bonded onto a rigid substrate using a thin layer of photoresist as an adhesive layer. Zinc patterns $60 \mu \mathrm{m}$ thick were electrodeposited through a photoresist mold via pulse reverse electroplating at an equivalent current density of $8 \mathrm{~mA} / \mathrm{cm}^{2}$. After the phororesist was removed, 
the metalized polymer film with microfabricated zinc patterns was separated from the rigid substrate (Figure 4(a-1)). Figure 5 shows images of the electroplated zinc inductor. PLA and PCL polymer films with designed thicknesses were prepared separately by solvent casting from dichloromethane. The PLA and PCL polymer films were cut to the appropriate designs and dimensions using a $\mathrm{CO}_{2}$ laser (Figure 4(a-2)).

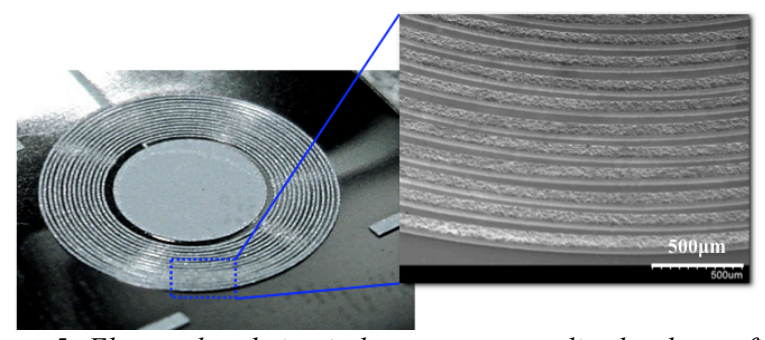

Figure 5: Electroplated zinc inductor onto metalized polymer film. The inset shows a scanning electron microscopy image of the electrodeposited zinc windings.

The flexible substrate with electroplated zinc patterns and a PLA polymer sheet fabricated as above with thicknesses ranging from 200 to $400 \mu \mathrm{m}$ were laminated under a 10-bar pressure at $160^{\circ} \mathrm{C}$ for 4 minutes. After lamination, the flexible polymer substrate was peeled off. The resulting structure consisted of the microfabricated zinc patterns embedded in a PLA polymer sheet. The remaining seed layer was removed by micro-polishing, limiting the number of wet processing steps (Figure 4(b)).

To define the sensor cavity, a biodegradable spacer consisting of a smaller PLA ring and a larger, concentrically-placed PCL ring was laminated onto the PLA sheet with embedded zinc microstructures (Figure 4(c)). A small amount of dichloromethane solvent was used as a bonding promoter. Then the PLA/Zn sheet was folded (Figure 4(d)) and laminated to form the 3-D PLA/Zn pressure sensor. The folding was performed with the assistance of selective heating along the folding line. The folding technique, adapted from [12], enables the microfabrication of a 3-D device with multiple metal layers and electrical interconnects using a single electrodeposition step. This is particularly favorable when the metallic and polymeric materials are sensitive to chemicals used in conventional microfabrication techniques. The lamination step to complete the pressure sensor fabrication was performed under pressure and at $55^{\circ} \mathrm{C}$. During the lamination, the PCL spacer softened and sealed the cavity. Finally, the device shape was defined around the inductor geometry by $\mathrm{CO}_{2}$ laser

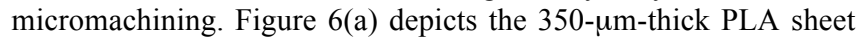
with embedded zinc patterns along with the spacers before folding and lamination. An example of a fully-fabricated and functional 10-mm-diameter device is shown in Figure 6(b). The design parameters are reported in Table 1.

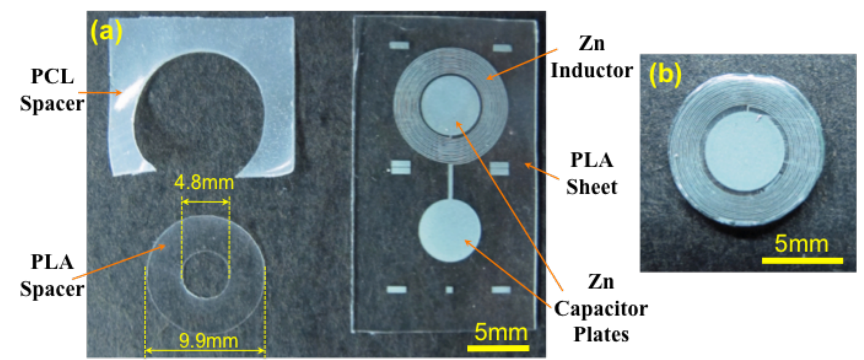

Figure 6: Microfabricated RF pressure sensor completely made of biodegradable materials, (a) before folding, and (b) after lamination and cutting.

\section{RESULTS}

Degradation Rate of Electroplated Zinc Samples

Zinc samples were electroplated for degradation tests in saline. The samples were between 1 and $2 \mathrm{~cm}^{2}$ in area and between 70 200 $\mu \mathrm{m}$ in thickness. The area and initial weight of the samples were measured after fabrication. Each specimen was immersed in $20 \mathrm{~mL}$ of Phosphate-Buffered Saline $1 \times$ (PBS buffer), and placed in an incubator at a temperature of $37 \pm 0.5^{\circ} \mathrm{C}$. Throughout a 30 -day timeframe, the weight of each specimen was measured daily. For daily weight measurements, the specimens were rinsed in deionized water and dried thoroughly. After weight measurement and before pursuing the experiment, the PBS buffer solution was refreshed by replacing half of it with fresh buffer.

The experimental results are plotted in Figure 7. The daily weight loss is normalized by the area of the $\mathrm{Zn}$ samples. The degradation rate of electroplated zinc was measured at $0.4 \mathrm{mg} /\left(\right.$ day $\left.\cdot \mathrm{cm}^{2}\right)$, which corresponds to approximately $0.6 \mu \mathrm{m} /$ day of degraded zinc material.

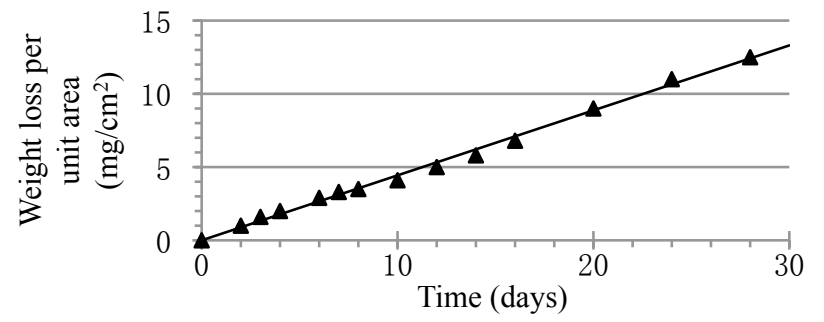

Figure 7: Biodegradability test result: daily weight loss per unit area of electroplated zinc in PBS solution.

\section{Zinc Inductor Characterization}

The inductance and quality factor of the microfabricated zinc inductor embedded in a PLA polymer layer was measured before folding using an impedance analyzer. The experimental results are presented in Figure 8. An inductance of $1.9 \mu \mathrm{H}$ was measured at frequencies below $50 \mathrm{MHz}$, with a quality factor exceeding 25 .

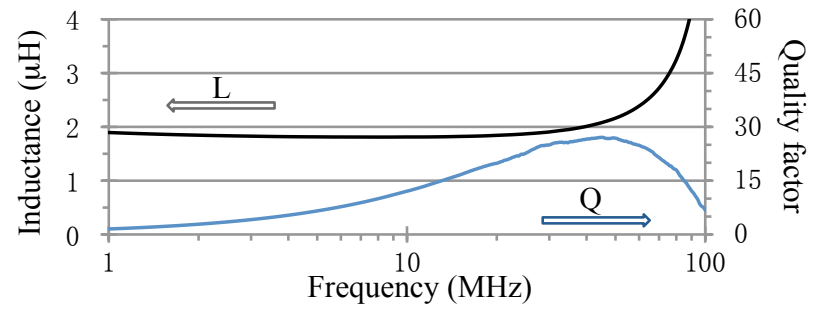

Figure 8: Measured inductance and quality factor of the electroplated zinc inductor embedded in a PLA polymer layer.

\section{Wireless Pressure Sensor Testing}

For wireless measurements, the fabricated sensor is coupled with an external coil (diameter $=1 \mathrm{~cm}, 5$ turns,). This coil has a $Q>200$ and a resonance above $100 \mathrm{MHz}$, and a coil to sensor distance of approximately $3 \mathrm{~mm}$. The impedance phase and magnitude are measured by an impedance analyzer as function of frequency. At the sensor resonance, changes in the external coil phase and magnitude are measured.

To determine the pressure sensitivity of the fabricated sensor, the sensor is placed in a sealed chamber. In these experiments, pressure was applied via an external air source. The experimental setup was shown in Figure 9. In operation, pressure is applied by turning on the air source while the valves 1 and 2 are left open. By closing valve 1 , the pressure in the chamber became stable and was measured by a pressure gauge. After electrical measurements, 
valve 2 was closed and valve 1 was re-opened as the air source pressure was modified. Valve 2 was then re-opened, and valve 1 was closed to repeat pressure and sensor electrical measurements over pressures ranging from 0 to $30 \mathrm{kPa}$. Aqueous environments could also be maintained in this apparatus by partially filling the sealed chamber containing the sensor with liquid.

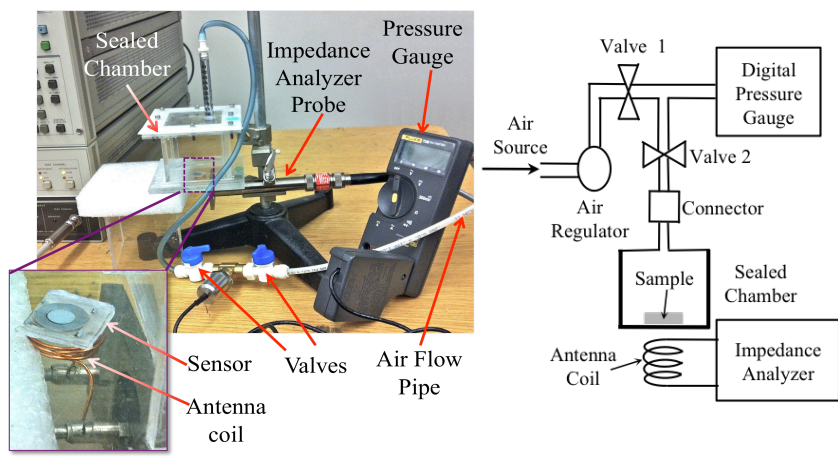

Figure 9: Pressure sensor test measurement setup (left) with schematic drawing (right).

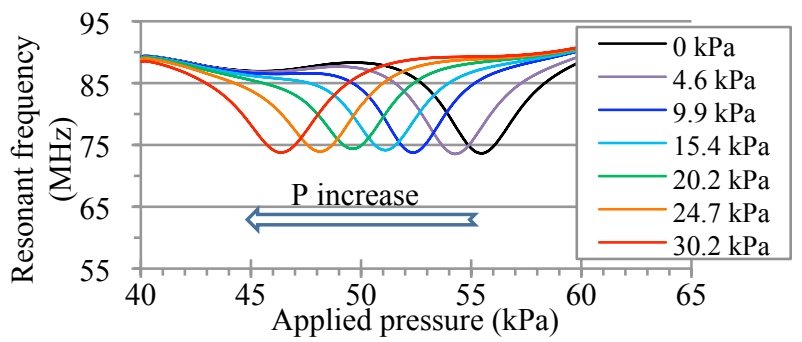

Figure 10: Impedance phase data as a function of frequency for several applied pressures.

The measured phase as a function of frequency for several applied pressures is shown in Figure 10. As the pressure increases, the minimum phase of the impedance shifts monotonically to lower frequencies because the deflection of the pressure sensitive $\mathrm{PLA} / \mathrm{Zn}$ sensor plates results in a capacitor gap decrease (i.e., a capacitance increase), and therefore a resonant frequency decrease. The impedance measurements for the microfabricated RF pressure sensor were repeated for several times under multiple pressure conditions. Figure 11 plots the resonant frequency of the sensor as a function of applied pressure in air and aqueous environments. A sensitivity of $-290 \mathrm{kHz} / \mathrm{kPa}$ was measured in air, with comparable sensitivity in water. This corresponds to a change in capacitance $\Delta \mathrm{C} / \mathrm{C}_{0}$ of $55 \%$. This sensitivity is approximately 6 times higher than that of the non-degradable implantable resonant pressure sensor in [2]. From the resonant frequency measurements, the calculated initial gap between the capacitor plates is approximately $40 \mu \mathrm{m}$, which is consistent with the expected value.

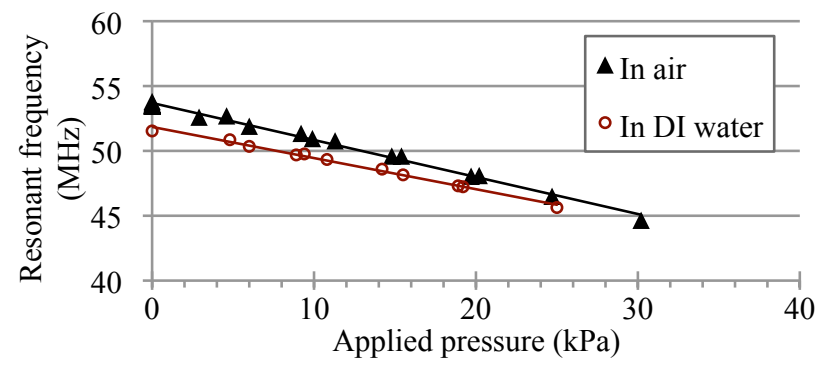

Figure 11: Resonant frequency of the sensor as a function of applied pressure in the sealed chamber.

\section{CONCLUSIONS}

A RF wireless LC resonant pressure sensor completely made of biodegradable materials was designed, microfabricated and characterized. Biocompatible zinc metal, which is compatible with MEMS fabrication techniques, was demonstrated to degrade under aqueous solutions. The zinc inductor and capacitor of the sensor were fabricated using MEMS technologies on flexible and chemically-resistant polymer and embedded in a pre-casted PLA film. The sensor was then fabricated by folding the PLA sheet with the embedded zinc microstructures. A biodegradable and adhesive spacer was utilized to form and seal the cavity during lamination.

After electrical characterization, the pressure sensor was experimentally tested in a sealed chamber with controlled pressure. The sensor response was wirelessly measured using an external coil connected to an impedance analyzer. The response time of the sensor to pressure change was instantaneous, and a sensor with sensitivity of $-290 \mathrm{kHz} / \mathrm{kPa}(-38 \mathrm{kHz} / \mathrm{mmHg})$ in air was measured experimentally. Comparable pressure-frequency behavior in an aqueous environment was observed. However, the functional lifetime of the sensors in the aqueous environment was shorter than predicted by degradation studies. These effects are currently under investigation.

\section{REFERENCES}

[1] R. S. Mackay et al., "Endoradiosonde", Nature, 197 (1957)

[2] M. A. Fonseca, J. Kroh, J. White, and M. G. Allen, "Flexible Wireless Passive Pressure Sensors for Biomedical Applications," Tech. Dig. Solid-State Sensor, Actuator, and Microsystems Workshop, Hilton Head, (2006)

[3] M. Wendlandt, F. Umbrecht, C. Hierold, F. Gattiker, J. Neuenschwander, and U. Sennhauser, "A Wireless Implantable Passive Strain Sensor (WIPSS)", European Symposium-Technical Aids for Rehabilitation, (2007)

[4] C.M. Boutry, H. Chandrahalim, and C. Hierold, "RF Conductivity of Biodegradable Conductive Polymers used for a New Generation of Partially/Fully Wireless Implantable Sensors", MEMS 2012, Paris, FRANCE, (2012)

[5] B. Finamore, G. Fedder, A. Khairi, J. Paramesh, L. Schultz, J. Burgess, P. Campbell, and L. Weiss, "Development of an Implantable Biodegradable Electrical Stimulator for Bone Repair", Biomedical Engineering Society Fall Meeting (2009)

[6] U. Edlund and A.-C. Albertsson, "Degradable Polymer Microspheres for Controlled Drug Delivery", Advances in Polymer Science, 157, (2002)

[7] R. Waksman, Biodegradable Stents: They Do Their Job and Disappear," Journal of Invasive Cardiol, 18, 2(2006)

[8] M. Schlesinger and M. Paunovic, "Modern Electroplating Fourth Edition", John Wiley \& Sons, Inc., 2000

[9] M.F. Ashby, "Engineering Materials and Processes Desk Reference", Elsevier, Inc., 2009

[10] O. I. Velikokhatnyi and P. N. Kumta, "First-principles studies on alloying and simplified thermodynamic aqueous chemical stability of calcium-, zinc-, aluminum-, yttrium- and iron-doped magnesium alloy", Acta Biomaterialia, 6, (2010)

[11] T. Kawamoto and T. Sugahara, "Examination of Properties of Bioabsorbable Osteosynthetic Material Using Finite Element Method", Journal of Hard Tissue Biology, 14, 2(2005)

[12] F. Herrault, S. Yorish, T.M. Crittenden, C.-H. Ji, and M.G. Allen, "Parylene-Insulated Ultradense Microfabricated Coils," Journal of Microelectromechanical Systems, 19, (2010)

\section{CONTACT}

*M. Luo, tel: +1-404-583-7312; mandyluo@gatech.edu 